\title{
That Guy There: The New Convention of the Populated Plan
}

\author{
DORA EPSTEIN JONES
}

$A+D$ Museum

Over-familiarity with the conventions of architectural representation in plan and perspective, paired with the advent of dimensional digital modeling software, has brought us to a new and now pervasive drawing convention: termed here as the "populated plan." First seen as a form of cartooning in the work of OMA, and then gradually made digital under BIG, MOS, Jimenez Lai and Andrew Kovacs (to name a few), the "populated plan" is a canny, perceptual machine, not just for viewing the spaces of architecture, but also for imagining the variety of activities that inhabit these spaces. The "populated plan" is a friendly kind of device, almost childlike in its Where's Waldo-wonderment, and filled with benign cars going to and fro, impromptu trees, lots of little people hard at work, a catalog of post-Ikea modern furnishings, and of course, plenty of mischief. "The populated plan" is a great way of enlivening an otherwise "boring" building using readily available software, and as such, it has become, very quickly, a popular choice among students of architecture, well, everywhere.

This paper examines the rise of what is rapidly becoming a new convention in architectural drawing. It tracks the brief history of the idea, and examines the relative ease of adaptability of this technique. In so doing, the paper describes the operations and mechanisms necessarily in play for a convention to become conventional. This paper also investigates the implications of the "populated plan" as a representational device, namely the scalar, social and political ramifications inscribed within. "Populated plans" are filled with representational people, conducting lives in orderly and disorderly ways. As such, "populated plans" readily accept the role of architecture as a spatially-loaded staging ground for social interactions, economic transactions, and political reactions. Far from the mute abstractions of the plan and section, the "populated plan" suggests architectural space as a space filled with human potential - some predictable, as in a Richard Scarry "What Do People Do All Day?" way, but more often, unpredictable, messy and hopeful. Finally, this paper asks us, as architectural instructors and critics to see the "populated plan" as far more than a passing fashion, and rather as a perfect product of a post-digital era of drawing one that derives its sensibilities from the perspectival sketch of old while exploiting the digital medium for its fineness of detail and richness of textural and color options. We must ask ourselves - is the "populated plan" a mere crutch, or can we insist that a new drawing convention carry human meaning, as it should?
In the beginning, there was the plan. It was abstract, ideal and good. If a man lay flat on his back, and a compass was placed at his navel, etc, etc, ...from Vitruvius on, we have had a particular confidence in the plan.

From Vitruvius on, or at least Alberti on, the plan has become the primary representational device of the architect. The plan is what the architect does, after all. As such, the plan, and all of its conventions hitherto, is also what we teach to future architects.

A plan has certain imperatives. It is normally imagined as a kind of horizontal section at about 4 feet from the floor, and therefore should use the line as a means to communicate the depth of the wall, including the ins and outs of niches and pilasters along that wall. The plan is also imagined as a spatial communicator - the area comprised by the boundary of the wall is understood as the area of the room, the perimeter of the volume, and/or moments of egress and spatial flow.

Plans, at least since Palladio, are also strikingly empty.

The scaling of the plan permits the imaginative speculation of occupation. A bedroom is envisioned as having a bed, for example. Sometimes, architects have drawn in furnishings to perhaps indicate placement. Plans almost always include sinks, baths and toilets, as these occupy the built space of the room. But otherwise, we have largely come to accept the role of the eye to "complete the spatial picture" or to imagine the modes, methods, number of occupants and types of occupation, dwelling, inhabitation and use once built.

The presence of the permissible scaling, or the specificity of the toilet, have kept the plan from total abstraction. The plan of the centrally-planned church as described in Alberti, and illustrated some 500 years later by Wittkower (via Bartoli), is a version of a totally abstract plan - a mere geometry of an angled polyhedron nearing the perfection of the circle down to the radius point 1 . In such total abstraction, the scaling is infinite, not measured. The human, at approximate 6 feet tall and 2 feet wide, could be squeezed or engulfed. The human does not really "matter" (which is odd given that the subject is Humanism).

Most plans are less abstract than Bartoli's polyhedron, and most are drawn to scale, but most plans are still very empty. 


\section{THE POPULATED PLAN}

Recently, the plan has paired with the advent of dimensional digital software to bring us a new architectural drawing convention, termed here as the "populated plan." First seen as a form of cartooning in the work of sketches of OMA, and then gradually made digital under BIG, MOS, Atelier Bow Wow, Jimenez Lai and Andrew Kovacs (to name a few), the "populated plan" is a canny, perceptual machine, not just for viewing the spaces of architecture, but also for imagining the variety of activities that inhabit these spaces. The "populated plan" is a friendly kind of device, almost childlike in its Where's Waldo-wonderment, and filled with benign cars going to and fro, impromptu trees, lots of little people hard at work, a catalog of post-lkea modern furnishings, and of course, plenty of mischief. "The populated plan" is a great way of enlivening an otherwise "boring" building using readily available software, and as such, it has become, very quickly, a popular choice among students of architecture, well, everywhere.

The provenance of a populated plan is more a mechanism of theory and presentation than it is a communicative device towards building. It is a mode of "selling a design" as a unified schema. It is not utilitarian, and as such, does not truly require exact scaling or exact measurement. Some populated plans are not plans at all but isometrics, axonometrics, and other sectional cuts. Some are aerial, some right at "eye level." Regardless of the style of plane or horizon, however, populated plans do serve to communicate spatial difference - inside and out - and spatial program - bathrooms, kitchens, dining rooms with chairs all askew, offices with papers, corridors with people passing, coffee counters down to the dirty cups.

The populated plan is the opposite of abstract. While informed by cartoon, the populated plan is still very much a drawing, and still fulfills the role of drawing as a representational medium. But, unlike the tipping point from plan to diagram, such as in the Wittkower example, or when Wittkower ultimately reduced the plans of the Palladian villa to a nine-square grid, the populated plan resists reduction. Populated plans thrive on addition. The visual interest is produced through multiplicity. The eye does not speculate spatial destiny, instead, the eye's attention is created through intense visual interest, the act of picking out objects and moments.

The exact ontological heritage of the populated plan has likely culprits. The following presents a few of the basic characteristics of the populated plan, as well as interpretive and speculative origins for each:

\section{a. Humor.}

The populated plan, presented as a line drawing of a field of spaces, objects and occupants, is perhaps birthed in Rem
Koolhaas' drawings for Parc de la Villette (1989) or in the earlier competition entry for Sante Gerasimos (1984). While more perspectival in nature than the populated plan will become, we can see in this drawing many of the features and advantages of populating. One is the tip of the hat to Archigram, and the "inflatable futures" of their cartoons. Another is the gentle, sunny-day, kite-flying dig at organized religion, in particular the campus of the monastery at Kefalonia.

For Koolhaas, the cloisters give way to concert venues and ferris wheels, agricultural duties to frisbees, gravestones to television sets. The monastery is recast as a pleasure field - at the center, a re-creation of the very diorama that the drawing produces. The plan here is drawn as a sloped axonometric more than as a perspective, and this lends itself to the whimsy of the content.

Of course, Archigram, the Smithsons and Venturi are the ancestors of this drawing, perhaps also Charles Moore and Cedric Price 2. But, unlike the ways in which these architects would either render scenes through cartooned perspectives, or use cartoon as a series of typological characters, Koolhaas' drawings represent a shift to an emphasis on spatial information for the entire scene. Indeed, the charm of the Koolhaas drawing is that it is much more like the whimsical tourist maps that impart information about way-finding as much as they delight in the silliness of ice cream at the mega-church. Historical information on this drawing suggests that Koolhaas was attempting to create a horizontal plane akin to his theorem of Delirious New York about the Downtown Athletic Club (eating oysters in boxing gloves...). In so doing, there is as much irony in the multiple event possibilities as there is a sense that the space of the image, its "picture plane," is a not a plan as much as an event horizon. Anything can happen, and haha, it does.

\section{b. Busy-ness.}

Populated plans are very busy drawings - lots of people doing different activities, lots of objects, improbable and not, and the communicated idea that the space represented is expansive enough to hold all of these people and things. Populated plans are a bit like Foucault's discussion of the "heterotopia," the cruise ship, the zoo, and therefore a plane that produces difference over sameness. The busy appearance of the populated plan enlivens the drawing a great deal - and thus moves the minimalist feel of the abstracted plan, or even the atmospheric rendering, into a series of non-abstract events.

Busy-ness is an emphatic quality in a number of projects, such as those by MOS, where the architecture is little more than building. MOS' entry for The Architectural Imagination is extremely busy - part of the delight, and part of the urban message, is based not on formal exuberance, but on the multiple lives of itty-bitty inhabitants. MOS even goes so far to 


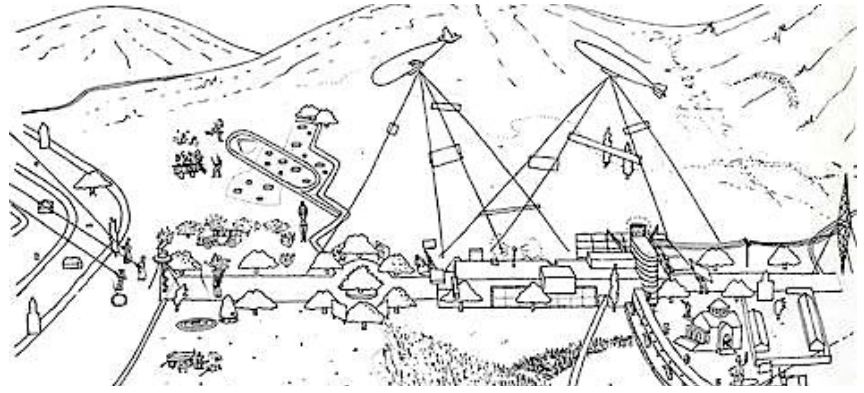

provide a full-color catalog of these folks, each with accessories and back-stories. The critique of architecture as the carrier for rejuvenated cityscapes or rehabilitated depressed urbanism is quite pointed in busy populated plans: rejuvenation and rehabilitation of urban scenes is ultimately not about the architecture but about masses and publics. In George Baird's essay on the Potteries Thinkbelt, he "accused" Price of having drawn people into the section as a kind of "crutch" to show how lively an otherwise undifferentiated mega-plane could be. The finger pointing was tender though, and tempered by a much more brutal attack on Saarinen's CBS building which Baird saw as so abstract as to be "antisemiotic." In this sense, "busy" is better than "empty" in that "busy" is super-articulate.

\section{c. Quilting.}

"Quilting" is an invented term to refer to a quality in the drawing technique itself. Quilting describes the cartoon quality that is produced through the even use of line weights. Each piece, regardless of mode of existence - wall, chair, person - is outlined with the same line thickness.

We can see this in evidence in as early as 2005 with Atelier Bow Wow's plan drawings, which are also likely culprits for the spreading popularity of this new convention.

Quilting is what makes the populated plan uniquely postdigital. Quilting does not belong to any one software empire. The line weights could be achieved in AutoCad as much as they could be achieved in Illustrator. Furthermore, since quilting could also refer to the use of multiple colors or the use of patterns and textures (like using a field of x's to color in a bush) within the outline of the figure, quilting could also be a product of Rhino. Quilting exemplifies a more casual and indifferent feeling for software in the production of a picture plane, and the ease in which the young architect (the student, especially) can move across platforms to satisfy each portion of the populated plan. Combined with the ability to zoom in and out with immediacy, the busy-ness of the drawing, once printed, is at times confused with the amount of detailing. Populated plans are delightful - but therein lies the rub.

\section{A VERY CONTEMPORARY SCENE}

In the writings of Alberto Perez-Gomez, we track the rise of the perspectival drawing that includes the presence of humans. They stand in front of facades as if standing in front of stage-set flats, looking fairly handsome and aloofly scientific $^{3}$. Even with articulated and delicate window pediments matching the articulated and delicate feathers in their tricorn hats, these men are merely geometric figures, intended to show the scale of the facade, the portico to the church, the expanse of the piazza. We see them 100 years later on grids that fade into the vanishing points of cities. 100 years after that, they stand under Viollet le Duc's vaults and Pugin's houses, maybe looking more forlorn.

The perspectival drawing in modernism is more flippant, and had a tendency to return the human to abstraction, especially if only a scalar figure. Broad-shouldered triangle-people pose Frenchly across Le Corbusier's cityscapes. Kahn liked a few people-ish characters here and there. Mostly though, the humans of modernism lounged around in drawings of city parks looking fairly blankly, in large part because they had no faces.

If humanism created "man as the measure," and then took that ideal rather literally, say under daVinci's Vitruvian Man, or completely abstractly, a la Bartoli, then modernism may be accused of having eliminated any one man in favor of a universal man, a modular man, the conformed citizen of the rational city. Today, the populated plan suggests a radical non-universality. "That guy there" is an extremely infinitesimal figure, located, placed, or missed entirely, in a complex field of multiple futures. What may begin as articulate and detailed, a way of showing multiplicity of spatial scenarios (as plans are wont to do!), is also a very serious proposal about contemporary subjectivity. In populated plans, humans are not there to show us the measure of the building, humans are there because buildings are participating in their very complicated and irreducible lives.

\section{THAT GUY THERE}

"Populated plans" are filled with representational people, conducting lives in orderly and disorderly ways. As such, "populated plans" readily accept the role of architecture as a spatially-loaded staging ground for social interactions, economic transactions, and political reactions. Far from the mute abstractions of the plan and section, the "populated plan" suggests architectural space as a space filled with human potential - some predictable, as in a Richard Scarry "What Do People Do All Day?" way, but more often, unpredictable, messy and hopeful. 
A new drawing convention in its nascency allows for caution. For the populated plan, even if it has evolved from cartoons, even if it's message is one of humor and high visual interest, does not mean that it is itself a benign instrument. The hyper-specificity of the populated plan should reflect the hyper-specifity of the human condition. "That guy there" is not a mere neutral signifier, but as stated previously, an articulated character. As such, it is incumbent upon us to reflect the diversity we wish there to be in the world.

Populated plans are delightful and alluring. For students of architecture, they may be a crutch for otherwise bland buildings, and given the relative skittishness of the software and the potency for praise for details, populated plans will only become more popular. As architectural instructors and critics, we need to see the populated plan as far more than a passing fashion. Populated plans may seem cartoonish and superficial, but as they move from trend to staple, we need to enunciate our demands. Populated plans should be considered with some ethical and moral weight - we might feel right about their illustrations of green practices or good parenting, but should we also not be wary of them showing the committing of crimes or the objectification of women? Can we dismiss the lives of the little characters with all of their papers and coffee cups as artistic vignettes, or is it time to impress upon our students, or anyone viewing these drawings that spaces and the people that inhabit those spaces, even speculatively, are far from neutral or silly? Carrying human meaning, in all of its intricacies, is light and heavy, clever and intense. It's not ideal, but it should be good.

\section{ENDNOTES}

1 See the polyhedron diagrams by Bartoli as illustrations in R. Wittkower, Architectural Principles in the Age of Humanism (WW Norton, 1971)

2 I would argue that the direct inheritance from Cedric Price is also informed by the way in which the Potteries Thinkbelt, for example, was envisioned as a plane of events. The most brilliant discussion of Price's ambitions in the drawing of the the Thinkbelt comes from G. Baird in "'La Dimension Amoureuse' in Architecture," C. Jencks \& G. Baird, Meaning in Architecture (Braziller, 1969)

3 In particular, Perez Gomez account of Chretien's physionotrace describes a sharp and tense moment between the presence of the figure and the illustration of scientific verification. See Variation 2 in Architectural Representation and the Perspective Hinge (MIT Press, 2000), 278.

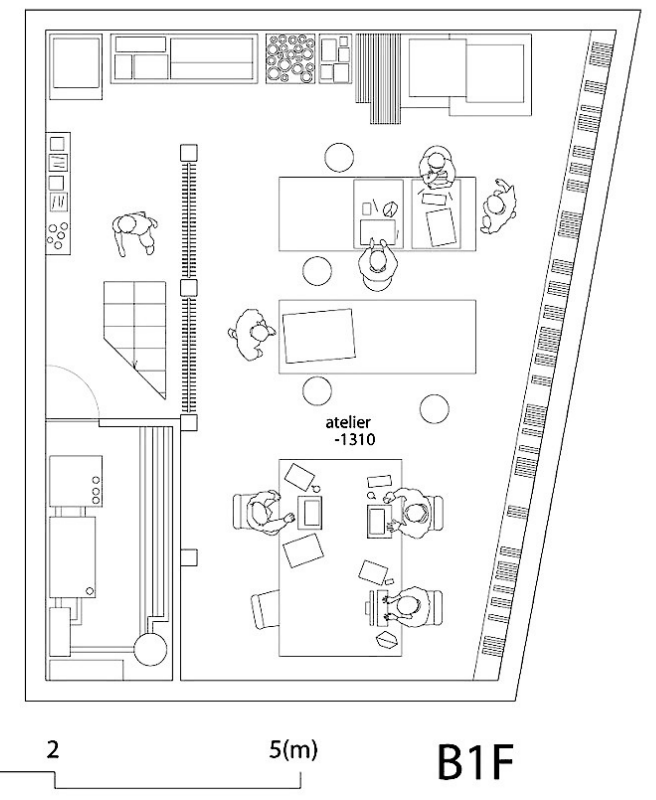

